\title{
CRECIMIENTO DE PLÁNTULAS DE NUEVE GENOTIPOS DE Bouteloua curtipendula CON DOS TAMAÑOS DE CARIÓPSIDE
}

\section{SEEDLING GROWTH OF NINE GENOTYPES OF Bouteloua curtipendula WITH TWO CARYOPSIS SIZES}

\section{J. Elvira Ramírez-Meléndez', Filogonio J. Hernández-Guzmán², Cándido López-Castañeda', Leonor Miranda-Jiménez ${ }^{1}$, Ma. de Jesús Carrillo-Llanos ${ }^{1}$ y Adrián R. Quero-Carrillo ${ }^{1}$ *}

\author{
'Colegio de Postgraduados, Postgrado en Ganadería, Campus Montecillo, Texcoco, Edo. México, México. ${ }^{2}$ Universidad Politécnica de Francisco I. \\ Madero, Tepatepec, Francisco I. Madero, Hidalgo, México.
}

\section{RESUMEN}

Establecer praderas en temporal árido representa un reto, por lo que un rápido desarrollo de la plántula implica ventajas. El presente estudio tuvo como objetivo evaluar el crecimiento inicial de Bouteloua curtipendula (Michx.) Torr. proveniente de dos tamaños de cariópside. El material vegetal incluyó a las variedades NdeM-125, NdeM-303, NdeM-417, NdeM-La Resolana y los genotipos CP-62 (Coahuila), CP-Centauro (Durango), CP-Teloxtoc (Puebla), CP-Ñañú1 (Hidalgo) y CP-Mixe (Oaxaca), con tamaños de cariópside grande (CG) y chico (CCh). Cariópsides de cada tamaño se sembraron en almácigo en sustrato de suelo, tepezil (mineral inerte y liviano que facilita la aireación y capilaridad) y corteza molida de árbol a razón 2:1:1. Se realizó trasplante en tubetes de $70 \mathrm{~cm}^{3} 21$ días después de siembra (dds) bajo un diseño completamente al azar. Se realizaron muestreos destructivos a los 43,56 , 67, 81 y 96 días después de siembra (dds) en condiciones de invernadero. Se determinó el peso de los componentes morfológicos en cada muestreo y se calculó la tasa absoluta de crecimiento (TAC), área foliar específica (AFE), tasa relativa de crecimiento (TRC) y tasa de asimilación neta (TAN). Se realizó análisis de varianza y prueba de comparación de medias de Tukey $(\mathrm{P}$ $\leq 0.05$ ). El rendimiento de materia seca (MS) de componentes morfológicos fue diferente $(P \leq 0.05)$ a 56 dds y se observó que sembrar CG en NdeMLa Resolana resultó en mayor MS aérea y radical y mostró mayor peso de inflorescencia $(P \leq 0.05)$ a 81 y 96 dds. Los valores máximos para TAN y TRC ocurrieron en etapas iniciales y disminuyeron a mayor edad de planta para todos los materiales. En TRC, NdeM-125 con CG fue mayor conforme avanzó la edad de la planta, mientras en TAN, CP-Ñañú1 mostró mayores valores ( $\mathrm{P} \leq$ 0.01). El AFE fue mayor para todos los materiales a 81 y 96 dds y CP-Mixe con CCh fue el mejor tratamiento. Seleccionar por tamaño de cariópside resulta en desarrollo diferencial de plántula. Las plantas de NdeM-La Resolana a partir de ambos tamaños de cariópside mostraron producción de semilla precoz, lo que resulta valioso para auto-recuperación de agostaderos.

Palabras clave: Bouteloua curtipendula, cariópsides clasificadas, crecimiento al establecimiento, desarrollo de plántula.

\section{SUMMARY}

Establishing rainfed grasslands under arid conditions represents a challenge; thus, rapid seedling development has advantages. This study aimed to evaluate the initial growth of Bouteloua curtipendula (Michx) Torr. coming from two sizes of caryopsis The plant material included the varieties NdeM-125, NdeM-303, NdeM-417, NdeM-La Resolana and genotypes CP-
62 (Coahuila), CP-Centauro (Durango), CP-Teloxtoc (Puebla), CP-Ñañú1 (Hidalgo) and CP-Mixe (Oaxaca) with large (LC) and small (SC) caryopsis sizes. Caryopses of each size were planted into seedbeds using soil, tepezil (an inert and light mineral that facilitates aeration and capillarity), and tree bark as substrate at a 2:1:1 ratio. The transplant was carried out into $70 \mathrm{~cm} 3$ tubes 21 days after sowing (DAS) under a completely randomized design Destructive sampling was performed at $43,56,67,81$ and 96 DAS under greenhouse conditions. The weight of a morphological components at each sampling was determined, and the absolute growth rate (AGR), specific foliar area (SFA), relative growth rate (RGR) and net assimilation rate (NAR) were calculated. Analysis of variance and Tukey mean comparison test $(P \leq 0.05)$ were performed. Dry matter (DM) production of morphological components was different $(\mathrm{P} \leq 0.05)$ at $56 \mathrm{DDS}$; also, planting LC of NdeM-La Resolana resulted in higher both aerial and root DM $(P \leq 0.05)$ and inflorescence weight at 81 and 96 DAS. The maximum values for NAR and RGR occurred at the initial stages and decreased as plants got older in all materials. For RGR, NdeM-125 SC was higher as the age of the plant advanced, while for NAR, $\mathrm{CP}$-Ñañu1 showed higher values $(\mathrm{P} \leq 0.01)$. SFA was highest for all materials at 81 and 96 DDS, and CP-Mixe with SC was the best treatment. Selection for caryopsis size results in differential seedling development. Plants of NdeM-La Resolana from both caryopsis sizes showed early seed production, which is valuable for self-recovering of rangelands.

Index words: Bouteloua curtipendula, classified caryopses, growth to establishment, seedling development.

\section{INTRODUCCIÓN}

Las zonas áridas y semiáridas en México representan más del $50 \%$ de territorio y éstas se dividen en dos tipos de vegetación, los matorrales que cubren $85 \%$ y los pastizales que representan $15 \%$, situación que era inversa hace 150 años (PMARP, 2012). El uso de gramíneas para la recuperación de la antigua condición y funcionalidad del pastizal representa una herramienta potencial para disminuir el avance de la desertificación al cubrir el suelo rápida y efectivamente (Sánchez-Arroyo et al., 2018) para su conservación ecológica y utilización bajo pastoreo, para lo cual es importante inducir un elevado vigor de planta y alta densidad de macollos mediante buen manejo (Quero 
et al., 2017a; Quero-Carrillo et al., 2014).

México es un centro de diversidad genética de muchas especies de pasto (Poaceae), entre ellas 57 del género Bouteloua, incluyendo al pasto Banderita (Bouteloua curtipendula), que se encuentra distribuida desde Canadá hasta Argentina (Peterson et al., 2015). Al respecto, se ha recolectado y evaluado germoplasma; sin embargo, esta actividad no se había realizado de manera sistemática (Morales et al., 2009). Para la rehabilitación de áreas degradadas de zonas semiáridas de México, Corrales et al. (2016) recomendaron el uso de especies nativas de pastos como Banderita, con el fin de no alterar el ecosistema de las zonas de escasa precipitación por ser tan vulnerable.

Para establecer praderas de temporal existen recomendaciones como usar las unidades de dispersión completas (glumas, lemas, paleas, aristas y ramillas modificadas) o cariópsides, otro factor a considerar es la latencia dada por barreras hormonales y físicas para imbibición y germinación (Quero-Carrillo et al., 2016); por lo anterior, recomendaron no eliminar brácteas florales en pastos nativos, mientras que en pastos introducidos sí es importante eliminar éstas para incrementar la germinación. En zonas semiáridas las siembras se realizan al establecimiento del temporal (julio) y dado que las temperaturas congelantes ocurren en octubre, los pastos tienen poco más de 90 días para establecerse, periodo en que la evaluación es importante.

El tamaño de cariópside ha sido importante para obtener, a través de mayor tamaño de embrión y reservas del endospermo, mayor peso y vigor de plántula en cultivos como maíz (García-Rodríguez etal., 2018) y en pastos como Lolium perenne L. (Smith et al., 2003) y pasto Banderita (Quero et al., 2017b) y mayor velocidad de germinación a diversas profundidades de siembra (Corrales et al., 2016). Por tanto, es importante evaluar el comportamiento de nuevas variedades y genotipos de pastos de zonas semiáridas de México y determinar su posible potencial de crecimiento los días iniciales después de la siembra para seleccionar por su aptitud competitiva de manera eficiente contra la maleza y sequía intraestival severa, entre otros aspectos que afectan su establecimiento en temporal semiárido.

El análisis de crecimiento vegetal usa mediciones directas como la materia seca y área foliar con la finalidad de describir crecimiento, lo que puede apoyar con mayor certidumbre la decisión de seleccionar genotipos para mejor establecimiento de praderas, ésto incluye: 1) tasa absoluta de crecimiento, definida como la capacidad vegetal para producir material nuevo en un tiempo determinado; Hunt (2017) y Barrera et al. (2010) la describieron como incremento en peso por unidad de tiempo; 2) tasa relativa de crecimiento, definida como la eficiencia para producir materia seca en un tiempo dado expresada en $\mathrm{g} \mathrm{g}^{-1} \mathrm{~d}^{-1}$ (Di Benedetto y Tognetti, 2016), mientras que James y Drenovsky (2007) la definieron como la capacidad de producir material nuevo a partir de una unidad de peso; 3) área foliar específica, la cual establece la relación entre área foliar y peso foliar como medida de abundancia foliar, ésta disminuye con la edad por remoción de carbohidratos solubles desde la hoja hacia los cariópsides (Pérez et al., 2004); 4) tasa de asimilación neta, definida como indicador de biomasa acumulada en función del aparato fotosintético y del tiempo (Álvarez-Holguín et al., 2017) o indicador de eficiencia del tejido asimilador de radiación solar para producir materia seca a través de fotosíntesis laminar (James, 2008). Los trabajos relacionados con las variables de crecimiento en pastos nativos como $B$. curtipendula con diferente tamaño de semilla son escasos; por lo tanto, el objetivo del presente estudio fue analizar el crecimiento de plántulas provenientes de dos tamaños de cariópside en materiales de esta especie.

\section{MATERIALES Y MÉTODOS}

\section{Sitio experimental y material genético}

El estudio se llevó a cabo en condiciones de invernadero en el Colegio de Postgraduados, Campus Montecillo, Texcoco, Estado de México, del 31 de mayo al 4 de septiembre de 2017. La temperatura del invernadero, determinada con data logger cada media hora, en promedio fue de $22.5^{\circ} \mathrm{C}$, máxima de $41^{\circ} \mathrm{C}$ y mínima de $13^{\circ} \mathrm{C}$.

Las semillas de B. curtipendula utilizadas provinieron de cosechas realizadas en 2016 (Cuadro 1), las cuales se mantuvieron en bolsas de manta en condiciones de laboratorio hasta su uso.

Cariópsides de cada material fueron obtenidos mediante la eliminación de brácteas accesorias por fricción física manual, con almohadilla y tapete corrugado; posteriormente, se separaron por tamaño con ayuda de tamices de $6 \mathrm{~mm}$ y se eliminaron cariópsides con cualquier daño físico mínimo visible; lo anterior, con auxilio de microscopio estereoscópico.

\section{Tratamientos, diseño y unidad experimental}

Los cariópsides se clasificaron por peso para cada genotipo. Se consideraron como cariópsides grandes aquellos en un intervalo de peso entre 0.0005 y $0.0010 \mathrm{~g}$ y como chicos a los inferior a $0.0005 \mathrm{~g}$. Los tratamientos incluyeron nueve materiales genéticos de $B$. curtipendula con los dos tamaños de cariópside: grande (CG) y chico 
Cuadro 1. Origen de la semilla de Bouteloua curtipendula (Michx.) Torr. utilizada en el estudio.

\begin{tabular}{lccc}
\hline Variedad/Genotipo & \multicolumn{1}{c}{ Identificación $^{+}$} & Lugar de cosecha & Fecha de cosecha \\
\hline NdeM-125 & SADER-SNICS: 1730 & Chilcuautla, Hidalgo & marzo de 2016 \\
Ndem-303 & SADER-SNICS: 1729 & Chilcuautla, Hidalgo & marzo de 2016 \\
NdeM-417 & SADER-SNICS: 1727 & Chilcuautla, Hidalgo & marzo de 2016 \\
NdeM-La Resolana & SADER-SNICS: 2164 & Chilcuautla, Hidalgo & marzo de 2016 \\
CP-62 & Chilcuautla, Hidalgo & marzo de 2016 \\
CP-Centauro & S. Juan del Río, Durango & octubre de 2016 \\
CP-Teloxtoc & Teloxtoc, Tehuacán, Puebla & octubre de 2016 \\
CP-Ñañú1 & Chilcuautla, Hidalgo & octubre de 2016 \\
CP-Mixe & Sierra Juárez, Oaxaca & octubre de 2016 \\
\hline
\end{tabular}

†Identificación Servicio Nacional de Inspección y Certificación de Semillas.

(CCh); es decir, 18 tratamientos con tres repeticiones bajo un diseño completamente al azar. Se consideró a una planta en tubete como la unidad experimental.

\section{Manejo del experimento}

Se utilizó un sustrato que incluyó suelo Vertisol del Valle del Mezquital de textura arcillosa, pH 6.9 y $2.4 \%$ de materia orgánica, además de grava yesosa o tepezil y corteza molida de árboles de la zona en proporción 2:1:1, el cual una vez mezclado se esterilizó en autoclave de fabricación propia con capacidad de $1 \mathrm{~m}^{3}$ durante $4 \mathrm{~h}$ a $121^{\circ} \mathrm{C}$. La siembra se realizó en sustrato húmedo el 31 de mayo de 2017 en charolas-almácigo de $30 \mathrm{~cm}$ de diámetro por $15 \mathrm{~cm}$ de profundidad con drenaje, y 21 días después se trasplantó una plántula por tubete de $70 \mathrm{~cm}^{3}$. Los riegos, tanto en charola como en tubete, fueron a saturación conforme a los requerimientos de la plántula. No se presentaron plagas ni enfermedades y no se fertilizó.

\section{Variables evaluadas}

Se realizaron muestreos destructivos a los $43,56,67$,
81 y 96 días después de la siembra (dds) en tres plantas por tratamiento. Las variables evaluadas para cada fecha de muestreo incluyeron área de las láminas foliares $\left(\mathrm{cm}^{2}\right)$ con integrador de área foliar marca Li-COR (Lincoln Nebraska, EUA), biomasa total (g MS/planta) incluyendo láminas foliares, raíces, tallos e inflorescencia, así como biomasa de las estructuras morfológicas por separado (g MS/planta); para ello, la planta completa se sacó de la maceta y se colocó en agua corriente hasta que el sustrato se desprendió de las raíces; posteriormente, la planta se separó en sus componente morfológicos: láminas foliares, tallos e inflorescencias. Se consideró a la corona como parte de la raíz y a las vainas como parte del tallo. Las estructuras morfológicas se colocaron en sobres de papel para su secado en estufa de aire forzado de $36 \mathrm{~L}$ de capacidad (Marca Dalvo, modelo Basic, Zelian S.A., Buenos Aires, Argentina) a $60^{\circ} \mathrm{C}$ durante $48 \mathrm{~h}$ (peso constante) y se pesó con balanza eléctrica de precisión $( \pm 0.001 \mathrm{~g})$ (Sartorius, modelo 1984, Bohemia, New York, EUA).

Adicionalmente se determinaron variables de crecimiento, por la diferencia en días entre cortes: tasa de crecimiento relativo (TRC), tasa de asimilación neta (TAN) y área foliar

Cuadro 2. Índices de crecimiento evaluados en materiales de Bouteloua curtipendula (Michx.) Torr.

\begin{tabular}{|c|c|c|c|}
\hline Índice & Fórmula & Unidad & Referencia \\
\hline TAC & $\left(P S_{2}-P S_{1}\right) /\left(T_{2}-T_{1}\right)$ & $\mathrm{g} \mathrm{d}^{-1}$ & (Hunt, 2003) \\
\hline TRC & $\left(\ln P S_{2}-\ln P S_{1}\right) /\left(T_{2}-T_{1}\right)$ & $\mathrm{g} \mathrm{g}^{-1} \mathrm{~d}^{-1}$ & (Di Benedetto y Tognetti, 2016) \\
\hline AFE & $\mathrm{AF} / \mathrm{PSH}$ & $\mathrm{cm}^{2} \mathrm{~g}^{-1}$ & (Pérez et al., 2004) \\
\hline TAN & {$\left[\left(\mathrm{PS}_{2}-\mathrm{PS}_{1}\right) /\left(\mathrm{T}_{2}-\mathrm{T}_{1}\right)\right] \times\left[\left(\ln A \mathrm{~F}_{2}-\ln A \mathrm{~F}_{1}\right) /\left(\mathrm{AF}_{2}-\mathrm{AF}_{1}\right]\right.$} & $\mathrm{g} \mathrm{cm}^{-2} \mathrm{~d}^{-1}$ & (Álvarez-Holguín et al., 2017) \\
\hline
\end{tabular}

TAC: tasa absoluta de crecimiento, TRC: tasa relativa de crecimiento, AFE: área foliar específica, TAN: tasa de asimilación neta, PS: peso seco planta completa, AF: área foliar, In: logaritmo natural, PSH: peso seco de hojas, T: tiempo (días), T1: medición inicial, $T_{2}$ : medición final en cada intervalo de tiempo. 
específica (AFE) (Cuadro 2). La TAN y AFE se obtuvieron con ayuda del peso de las láminas foliares y su área $\left(\mathrm{cm}^{2}\right)$.

\section{Análisis estadístico}

Los datos se analizaron con el paquete estadístico SAS versión 9.2 (SAS Institute, 2009) y la prueba de Tukey ( $\mathrm{P} \leq$ 0.05) para separación de medias.

\section{RESULTADOS Y DISCUSIÓN}

\section{Biomasa total}

Aunque se observó diferencia significativa en peso de componentes morfológicos a partir de los 56 dds y total a los 43 dds ( $\leq \leq 0.001$; Cuadro 3), CP-Mixe y CP-Ñañú1 de CG mostraron más de $0.02 \mathrm{~g}$ por planta, junto con NdeM-La Resolana de CCh, lo anterior es importante dado que, a mayor crecimiento a temprana edad, hay mayor oportunidad competitiva ante la maleza y sequía intraestival, irregular y sempiterna en siembras de temporal en zonas áridas y semiáridas. La variedad NdeM-La Resolana en CG acumuló mayor biomasa total y fue predominante a partir de 67 dds.

La influencia de tamaño de cariópside en el desarrollo de plántulas ha sido discutida por varios autores, en pastos como Lolium perenne (Smith et al., 2003) y nativos de México (Quero et al., 2017b), quienes mencionan que a mayor tamaño de cariópside se alcanza mayor tamaño de raíz y parte aérea en menor tiempo; lo anterior se observó en el presente experimento, con excepción de NdM-125, donde CCh fue más competitivo con respecto a CG; sin embargo, predomina el efecto del CG sobre el desarrollo en el resto de los materiales evaluados.

\section{Composición morfológica}

Se observaron diferencias significativas $(P \leq 0.05)$ por fecha para componentes morfológicos y tamaño de

Cuadro 3. Biomasa total ( $\mathrm{g} /$ planta) en muestreos destructivos de nueve genotipos de Bouteloua curtipendula (Michx.) Torr., desarrollados en condiciones de invernadero.

\begin{tabular}{|c|c|c|c|c|c|c|c|}
\hline \multirow[b]{2}{*}{ Genotipos } & \multirow[b]{2}{*}{$\mathrm{T}$} & \multicolumn{5}{|c|}{ dds } & \multirow[b]{2}{*}{ Total } \\
\hline & & 43 & 56 & 67 & 81 & 96 & \\
\hline \multirow{2}{*}{ NdeM-125 } & CG & 0.0062 & $0.021 \mathrm{c}$ & $0.036 \mathrm{e}$ & $0.108 \mathrm{f}$ & $0.323 \mathrm{de}$ & $0.495 f$ \\
\hline & CCh & 0.0085 & $0.126 a b$ & $0.212 \mathrm{abc}$ & $0.310 a b c$ & $0.467 \mathrm{bc}$ & $1.124 b$ \\
\hline \multirow{2}{*}{ NdeM-417 } & CG & 0.0083 & $0.081 \mathrm{bc}$ & $0.121 \mathrm{cde}$ & $0.187 \mathrm{ef}$ & $0.290 \mathrm{e}$ & 0.687 cde \\
\hline & CCh & 0.0075 & $0.057 \mathrm{bc}$ & $0.087 \mathrm{de}$ & $0.169 \mathrm{ef}$ & $0.273 \mathrm{e}$ & 0.594 ef \\
\hline \multirow{2}{*}{ NdeM-303 } & $\mathrm{CG}$ & 0.0050 & $0.049 \mathrm{bc}$ & $0.103 \mathrm{de}$ & 0.167 ef & 0.375 cde & 0.698 cde \\
\hline & CCh & 0.0125 & 0.048 bc & $0.104 \mathrm{de}$ & $0.184 \mathrm{ef}$ & $0.310 \mathrm{de}$ & 0.659 def \\
\hline \multirow{2}{*}{$\begin{array}{l}\text { NdeM-La } \\
\text { Resolana }\end{array}$} & CG & 0.0093 & $0.105 a b$ & $0.262 \mathrm{a}$ & $0.384 a$ & $0.853 a$ & $1.614 a$ \\
\hline & CCh & 0.0200 & 0.078 bc & $0.131 \mathrm{~cd}$ & $0.164 \mathrm{ef}$ & $0.416 \mathrm{bcd}$ & $0.809 \mathrm{~cd}$ \\
\hline \multirow{2}{*}{ CP-62 } & CG & 0.0128 & 0.072 bc & 0.114 de & $0.215 \mathrm{de}$ & $0.335 \mathrm{de}$ & 0.748 cde \\
\hline & CCh & 0.0152 & 0.074 bc & $0.149 \mathrm{bcd}$ & $0.283 \mathrm{bcd}$ & $0.334 \mathrm{de}$ & 0.855 c \\
\hline \multirow{2}{*}{ CP-Centauro } & CG & 0.0189 & $0.084 \mathrm{bc}$ & $0.144 \mathrm{~cd}$ & 0.240 cde & $0.339 \mathrm{de}$ & $0.826 \mathrm{~cd}$ \\
\hline & CCh & 0.0157 & $0.057 \mathrm{bc}$ & $0.106 \mathrm{de}$ & $0.205 \mathrm{de}$ & $0.294 \mathrm{e}$ & 0.677 cde \\
\hline \multirow{2}{*}{ CP-Teloxtoc } & CG & 0.0064 & $0.100 a b c$ & $0.209 a b c$ & $0.339 a b$ & $0.501 b$ & 1.156 b \\
\hline & $\mathrm{CCh}$ & 0.0047 & 0.080 bc & $0.168 \mathrm{abcd}$ & $0.297 \mathrm{abcd}$ & $0.500 \mathrm{~b}$ & $1.050 \mathrm{~b}$ \\
\hline \multirow{2}{*}{ CP-Ñañú1 } & CG & 0.0229 & $0.087 a b c$ & 0.127 cde & $0.185 \mathrm{ef}$ & 0.372 cde & $0.794 \mathrm{~cd}$ \\
\hline & CCh & 0.0058 & 0.061 bc & $0.083 \mathrm{de}$ & $0.163 \mathrm{ef}$ & $0.296 \mathrm{e}$ & 0.609 ef \\
\hline \multirow{2}{*}{ CP-Mixe } & CG & 0.0236 & $0.169 a$ & $0.227 \mathrm{ab}$ & $0.328 a b c$ & $0.414 \mathrm{bcd}$ & $1.161 \mathrm{~b}$ \\
\hline & CCh & 0.0144 & 0.077 bc & $0.114 \mathrm{de}$ & 0.252 bcde & $0.334 \mathrm{de}$ & $0.791 \mathrm{~cd}$ \\
\hline DSH (0.05) & & 0.0198 & 0.0816 & 0.0944 & 0.0927 & 0.1148 & 0.1796 \\
\hline Significancia & & NS & $\star *$ & $\star \star$ & $* *$ & $\star *$ & $\star *$ \\
\hline
\end{tabular}

Letras iguales por columna indican promedios estadísticamente iguales (Tukey, P $\leq 0.05)$. T: tamaño de cariópside, dds: días después de siembra CG: tamaño de cariópside grande, CCh: tamaño de cariópside chico, *: P $\leq 0.05, * *: P \leq 0.01$, NS: no significativo, DSH: diferencia significativa honesta. 
cariópside a 56, 67, 81 y 96 dds. A los 43 dds, destacó el genotipo CP-Mixe en CG al mostrar mayor biomasa de hoja, raíces y total $(P \leq 0.01)$; similarmente, para el último muestreo (96 dds), solamente en biomasa de raíz no fue diferente al genotipo NdeM-La Resolana en CG y CCh, los cuales fueron superiores. Por otro lado, el CG fue determinante para que NdeM-La Resolana a 56 y 96 dds produjera mayor MS $(P<0.05)$, mayor biomasa de láminas foliares y raíz; similarmente, desde 81 dds, las plantas originadas de CG mostraron tejido de inflorescencia (Figura 1); al respecto, Castañeda-Saucedo et al. (2009) indicaron al tamaño de semilla como fundamental en el desarrollo de cultivos básicos como cebada y trigo.

Alcanzar la madurez fisiológica a temprana edad de planta (producción de semilla el mismo año de siembra) es importante en praderas de temporal, debido a que en dichas condiciones de temporal en combinación con latencia de la semilla, las praderas siguen mostrando plántulas al segundo y tercer año después de siembra y se considera que éstas alcanzan su mayor densidad poblacional y productividad al tercer año (Quero-Carrillo et al., 2014). Se ha demostrado que en siembras con cariópsides grandes en pasto Banderita se presenta mayor velocidad de germinación y emergencia de plántulas (Hernández-Guzmán et al., 2015; Quero et al., 2017a); por tanto, la producción temprana de semilla indica mayor capacidad de establecimiento y cobertura potencial del agostadero, dado que al siguiente periodo de lluvias puede ocurrir germinación de semilla al año de establecimiento, atributo encontrado únicamente en NdeM-La Resolana. Las plantas de CG asignaron mayor cantidad de asimilados al desarrollo de la parte aérea con respecto a la raíz en comparación con lo sucedido en plantas generadas a partir de CCh.

En promedio, los materiales de CCh resultaron con menor biomasa total (Figura 1a). Durante etapas tempranas (43 y 56 dds), las plantas de NdeM-La Resolana, como genotipo de mayor producción, no mostraron grandes diferencias en biomasa total por efecto del tamaño de cariópside; sin embargo, las plantas originadas de CG mostraron consistentemente mayor asignación de fotosintatos hacia la parte aérea con respecto a la raíz: 0.49, 0.60, 0.5, 0.4 y $0.37 \mathrm{~g}$, respectivamente, para cada fecha de muestreo en este material (Figura 1b). Esta relación se mantuvo hasta los últimos cuatro muestreos y fue superior a la obtenida con la utilización de CG.

Al inicio de los muestreos, los materiales con mayor rendimiento de biomasa total (CP-Mixe-CG; $0.0236 \mathrm{~g} /$ planta) asignaron mayores recursos al desarrollo de

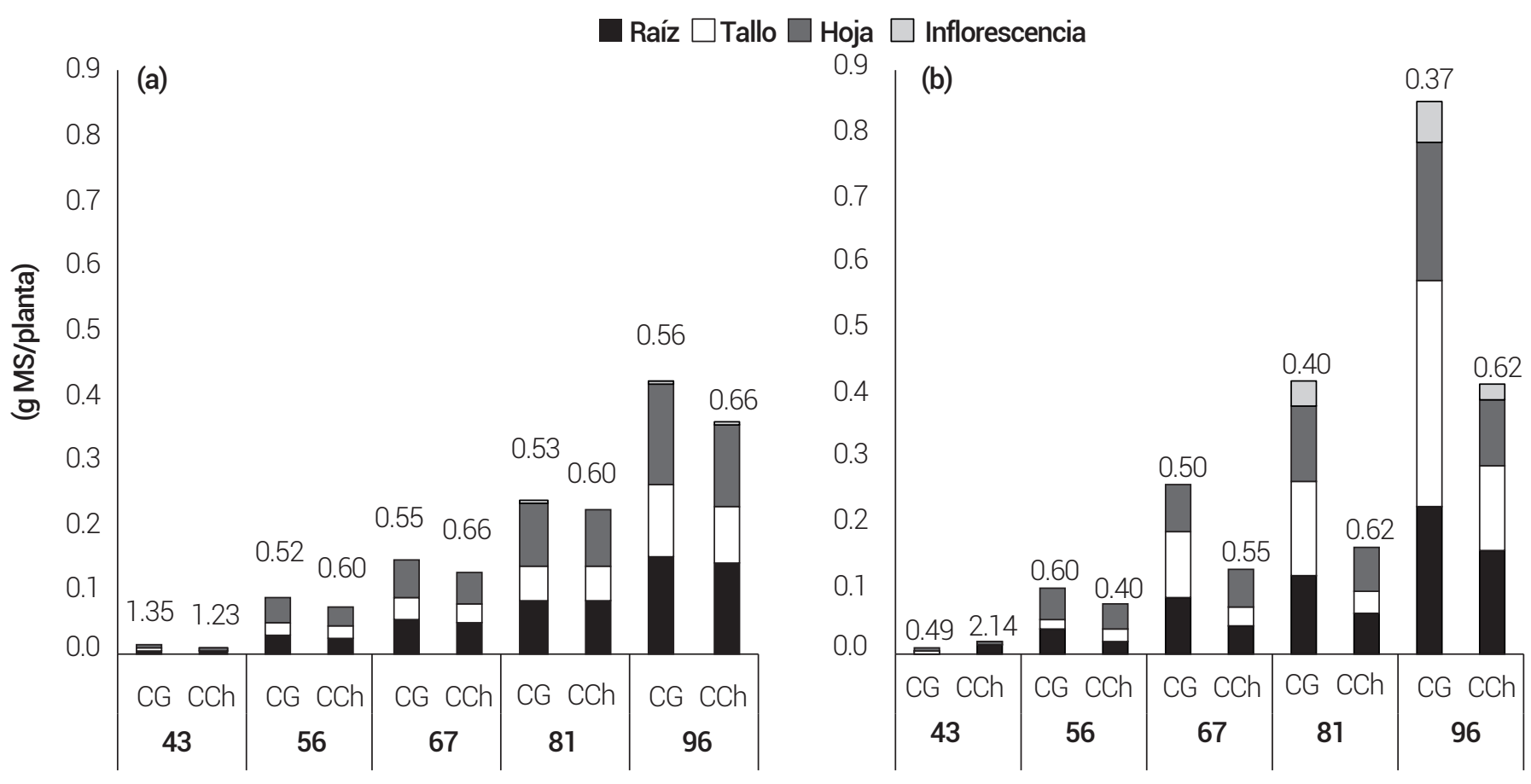

Tamaños de cariópside y tiempos de muestreo

Figura 1. Composición morfológica de materiales de Bouteloua curtipendula (a) y la variedad NdeM-La Resolana (b) de cariópsides grandes (CG) y chicos (CCh) en muestreos destructivos secuenciales bajo condiciones de invernadero. Valores sobre las barras indican la relación raíz:parte aérea. 
Cuadro 4. Tasa absoluta de crecimiento $\left(\mathrm{g} \mathrm{d}^{-1}\right)$ en genotipos de Bouteloua curtipendula provenientes de cariópside de diferente tamaño a través de muestreos (dds) en invernadero.

\begin{tabular}{|c|c|c|c|c|c|}
\hline \multirow{2}{*}{ Genotipos } & \multirow{2}{*}{$\begin{array}{l}\text { Tamaño de } \\
\text { cariópside }\end{array}$} & \multicolumn{4}{|c|}{ Muestreos (dds) } \\
\hline & & 56 & 67 & 81 & 96 \\
\hline \multirow{2}{*}{ NdeM-125 } & G & $0.0011 \mathrm{c}$ & 0.0015 & 0.0056 bcde & $0.0153 \mathrm{bc}$ \\
\hline & $\mathrm{Ch}$ & $0.0084 a b$ & 0.0095 & 0.0076 abcde & $0.0112 \mathrm{bcd}$ \\
\hline \multirow{2}{*}{ NdeM-417 } & G & $0.0056 a b c$ & 0.0040 & 0.0051 bcde & $0.0074 \mathrm{bcd}$ \\
\hline & $\mathrm{Ch}$ & $0.0039 \mathrm{bc}$ & 0.0030 & 0.0063 bcde & 0.0074 bcd \\
\hline \multirow{2}{*}{ NdeM-303 } & G & 0.0035 bc & 0.0053 & 0.0047 cde & 0.0149 bc \\
\hline & $\mathrm{Ch}$ & 0.0027 bc & 0.0056 & 0.0059 bcde & $0.0090 \mathrm{bcd}$ \\
\hline \multirow{2}{*}{$\begin{array}{l}\text { NdeM-La } \\
\text { Resolana }\end{array}$} & G & $0.0074 a b c$ & 0.0157 & $0.0123 a$ & 0.0308 a \\
\hline & $\mathrm{Ch}$ & 0.0044 bc & 0.0054 & 0.0025 e & $0.0180 \mathrm{~b}$ \\
\hline \multirow{2}{*}{ CP-62 } & G & $0.0045 b c$ & 0.0042 & 0.0077 abcde & $0.0086 \mathrm{bcd}$ \\
\hline & $\mathrm{Ch}$ & $0.0050 a b c$ & 0.0068 & $0.0103 a b c$ & $0.0036 d$ \\
\hline \multirow{2}{*}{ CP-Centauro } & G & $0.0050 a b c$ & 0.0060 & 0.0074 abcde & $0.0071 \mathrm{~cd}$ \\
\hline & $\mathrm{Ch}$ & 0.0032 bc & 0.0049 & 0.0076 abcde & $0.0064 \mathrm{~cd}$ \\
\hline \multirow{2}{*}{ CP-Teloxtoc } & G & $0.0072 a b c$ & 0.0109 & $0.0100 \mathrm{abcd}$ & $0.0116 \mathrm{bcd}$ \\
\hline & $\mathrm{Ch}$ & $0.0058 a b c$ & 0.0088 & $0.0099 \mathrm{abcd}$ & $0.0145 b c$ \\
\hline \multirow{2}{*}{ CP-Ñañú1 } & G & $0.0049 a b c$ & 0.0039 & 0.0045 de & $0.0134 \mathrm{bcd}$ \\
\hline & $\mathrm{Ch}$ & $0.0042 b c$ & 0.0022 & 0.0061 bcde & $0.0095 \mathrm{bcd}$ \\
\hline \multirow{2}{*}{ CP-Mixe } & G & $0.0111 \mathrm{a}$ & 0.0059 & 0.0077 abcde & $0.0062 \mathrm{~cd}$ \\
\hline & $\mathrm{Ch}$ & $0.0048 a b c$ & 0.0037 & $0.0106 a b$ & $0.0059 \mathrm{~cd}$ \\
\hline DSH (0.05) & & 0.0066 & 0.0155 & 0.0057 & 0.0107 \\
\hline Significancia & & $\star \star$ & NS & $\star \star \star ~$ & $\star \star \star$ \\
\hline
\end{tabular}

Letras iguales por columna indican medias estadísticamente similares $(\mathrm{P} \leq 0.05)$. dds: días después de la siembra, G: tamaño de cariópside grande, Ch: tamaño de cariópside chico, $* *: P \leq 0.01, * * *: P \leq 0.001, D S H$ : diferencia significativa honesta, NS: no significativo.

raíz que a la hoja, mientras que el genotipo de mayor rendimiento de biomasa al final del experimento (NdeMLa Resolana-CG) mostró menor relacion raíz:parte aérea (R:PA), lo que indica que se asignaron más fotosintatos a la hoja y tallo con R:PA de 0.37 (Figura 1b), no así para CCh donde se derivó mayor proporción a la raíz, misma que se mantuvo durante el periodo de evaluacion (0.62, Figura $1 b)$; lo anterior es valioso para seleccionar para capacidad de establecimiento.

\section{Índices de crecimiento vegetal}

La TAC expresa la velocidad de incremento de MS en pasto Banderita; Álvarez-Holguín et al. (2017) encontraron valores de $0.458 \mathrm{~g} \mathrm{~d}^{-1}$ en condiciones de invernadero; no existen en la literatura reportes de este indicador a partir de plantas clasificadas por tamaño de cariópside. En el presente estudio se observaron diferencias de 43 a 56 dds para TAC ( $P \leq 0.01$; Cuadro 4), donde CP-Mixe con CG mostró valores superiores $\left(0.0111 \mathrm{~g} \mathrm{~d}^{-1}\right)$; sin embargo, no se observó así para el intervalo de 57 a 67 dds, en el cual ningún material fue diferente $(P>0.05)$.

En el intervalo de 68 a 81 dds el genotipo NdeM-La Resolana de CG mostró mayor TAC $\left(0.0123 \mathrm{~g} \mathrm{~d}^{-1}\right)$ y continuó con este comportamiento hasta el último intervalo, de 82 a 96 dds ( $P \leq 0.001$ ); lo anterior puede estar relacionado con el inicio de incremento importante en el desarrollo de biomasa radical (Figura 1b) a partir de esta edad, lo que indica la consolidación de la biomasa aérea para apoyar el desarrollo de la planta, ya que tanto CP-Mixe como NdeM-La Resolana de CG presentaron mayor TAC ( $\leq 0.01$; Cuadro 4), al igual que tejido de raíces, como fue consignado por Cruz et al. (2017), quienes mencionaron que plantas eficientes invierten mayor parte de su crecimiento en expandir al área foliar y mejorar la captación de radiación solar. 
La TRC indica la capacidad de la planta para producir material nuevo; al respecto, en maíz (Zea mays L.) Rincón et al. (2007) reportaron valores de $0.01 \mathrm{~g} \mathrm{~g}^{-1} \mathrm{~d}^{-1}$ a los 55 dds. En el presente estudio se observaron diferencias significativas solamente en el intervalo de 68 a 81 dds ( $P$ $\leq 0.001)$ y de 82 a 96 dds $(P \leq 0.01$; Cuadro 5$)$. NdeM-125 con CG mostró los valores mayores (0.0846 y $0.0781 \mathrm{~g} \mathrm{~g}^{-1}$ $\left.\mathrm{d}^{-1}\right)$. El resto de los genotipos mostró valores elevados en los primeros días, de 43 a 56 y 57 a 67; sin embargo, no fueron significativos $(P>0.05)$; lo anterior puede deberse a factores que determinan el cálculo de la TRC como limitado crecimiento radical debido al tamaño limitado del contenedor, calidad de sustrato para liberar el máximo tejido subterráneo por muestreo, entre otros.

Los valores de TRC son mayores en los primeros estadios de crecimiento y disminuyen conforme la planta aumenta de tamaño (Di Benedetto y Tognetti, 2016). Reich et al. (2003) reportaron en pasto Banderita valores de 0.105 $\mathrm{g} \mathrm{g}^{-1} \mathrm{~d}^{-1}$, similares a los encontrados en el presente estudio a los 43 dds. Valores altos en TRC se relacionan con incremento en TAN para NdeM-125 de CG $\left(0.0055 \mathrm{~g} \mathrm{~cm}^{-2}\right.$ $\mathrm{d}^{-1}$ ), ambos índices proporcionan evidencias para evaluar el crecimiento de la planta entera y sus componentes (Fernández et al., 2002). En este sentido, valores altos en TRC indican un comportamiento similar en TAN debido al desarrollo del aparato asimilador.

Una elevada AFE puede indicar mayor valor nutritivo; en pasto Mulato (Brachiaria cv. híbrido) el valor más alto en AFE $\left(0.016 \mathrm{~m}^{2} \mathrm{~kg}^{-1}\right)$ se observó a los 28 dds (Pérez et al., 2004); en el presente estudio, los valores máximos se alcanzaron después de 68 dds, CP-Mixe CCh mostró un incremento y fue predominante a $81 \mathrm{dds}$, con $151.88 \mathrm{~cm}^{2}$

Cuadro 5. Tasa relativa de crecimiento $\left(\mathrm{g} \mathrm{g}^{-1} \mathrm{~d}^{-1}\right)$ en genotipos de Bouteloua curtipendula (Michx.) Torr. provenientes de cariópside de diferente tamaño a través de muestreos, en invernadero.

\begin{tabular}{|c|c|c|c|c|c|}
\hline \multirow{2}{*}{ Genotipos } & \multirow{2}{*}{$\begin{array}{l}\text { Tamaño de } \\
\text { cariópside }\end{array}$} & \multicolumn{4}{|c|}{ Muestreos (dds) } \\
\hline & & 56 & 67 & 81 & 96 \\
\hline \multirow{2}{*}{ NdeM-125 } & G & 0.0940 & 0.0541 & $0.0846 a$ & $0.0781 \mathrm{a}$ \\
\hline & $\mathrm{Ch}$ & 0.2173 & 0.0598 & 0.0294 bc & $0.0292 \mathrm{bcd}$ \\
\hline \multirow{2}{*}{ NdeM-417 } & $G$ & 0.1783 & 0.0411 & $0.0330 \mathrm{bc}$ & $0.0320 \mathrm{bcd}$ \\
\hline & $\mathrm{Ch}$ & 0.1790 & 0.0428 & $0.0512 a b c$ & $0.0341 \mathrm{bcd}$ \\
\hline \multirow{2}{*}{ NdeM-303 } & G & 0.1886 & 0.0725 & 0.0371 bc & $0.0580 a b c$ \\
\hline & $\mathrm{Ch}$ & 0.1154 & 0.0767 & 0.0444 bc & $0.0370 \mathrm{abcd}$ \\
\hline \multirow{2}{*}{$\begin{array}{l}\text { NdeM-La } \\
\text { Resolana }\end{array}$} & G & 0.1857 & 0.0910 & 0.0377 bc & $0.0503 \mathrm{abcd}$ \\
\hline & $\mathrm{Ch}$ & 0.1062 & 0.0523 & $0.0170 \mathrm{c}$ & $0.0665 a b$ \\
\hline \multirow{2}{*}{ CP-62 } & G & 0.1373 & 0.0463 & $0.0488 a b c$ & $0.0318 \mathrm{bcd}$ \\
\hline & $\mathrm{Ch}$ & 0.1370 & 0.0600 & $0.0504 a b c$ & $0.0118 d$ \\
\hline \multirow{2}{*}{ CP-Centauro } & G & 0.1158 & 0.0535 & $0.0395 \mathrm{bc}$ & $0.0248 \mathrm{bcd}$ \\
\hline & $\mathrm{Ch}$ & 0.0996 & 0.0595 & $0.0508 a b c$ & $0.0271 \mathrm{bcd}$ \\
\hline \multirow{2}{*}{ CP-Teloxtoc } & G & 0.2103 & 0.0826 & 0.0376 bc & $0.0281 \mathrm{bcd}$ \\
\hline & $\mathrm{Ch}$ & 0.2174 & 0.0824 & $0.0441 \mathrm{bc}$ & $0.0372 \mathrm{abcd}$ \\
\hline \multirow{2}{*}{ CP-Ñañú1 } & G & 0.1065 & 0.0375 & 0.0286 bc & $0.0502 \mathrm{abcd}$ \\
\hline & $\mathrm{Ch}$ & 0.1810 & 0.0319 & $0.0518 a b c$ & $0.0426 \mathrm{abcd}$ \\
\hline \multirow{2}{*}{ CP-Mixe } & G & 0.1520 & 0.0302 & $0.0282 \mathrm{bc}$ & $0.0167 \mathrm{~cd}$ \\
\hline & $\mathrm{Ch}$ & 0.1292 & 0.0393 & $0.0608 a b$ & $0.0202 \mathrm{~cd}$ \\
\hline DSH (0.05) & & 0.163 & 0.127 & 0.037 & 0.0422 \\
\hline Significancia & & NS & NS & $\star \star \star ~$ & $\star *$ \\
\hline
\end{tabular}


Cuadro 6. Área foliar específica $\left(\mathrm{cm}^{2} \mathrm{~g}^{-1}\right)$ en genotipos de Bouteloua curtipendula (Michx.) Torr., provenientes de cariópsides de diferente tamaño a través de muestreos, en invernadero.

\begin{tabular}{lccccc}
\hline \multirow{2}{*}{ Genotipos } & Tamaño de & \multicolumn{4}{c}{ Muestreos (dds) } \\
\cline { 2 - 5 } NdeM-125 & cariópside & 56 & 67 & 81 & 96 \\
& $\mathrm{G}$ & $1.76 \mathrm{ab}$ & $1.86 \mathrm{~b}$ & $110.48 \mathrm{ab}$ & 95.05 \\
NdeM-417 & $\mathrm{G}$ & $2.66 \mathrm{ab}$ & $6.54 \mathrm{ab}$ & $115.29 \mathrm{a}$ & 105.43 \\
& $\mathrm{G}$ & $9.87 \mathrm{a}$ & $9.92 \mathrm{ab}$ & $76.15 \mathrm{ab}$ & 101.60 \\
NdeM-303 & $\mathrm{Ch}$ & $0.38 \mathrm{~b}$ & $1.74 \mathrm{~b}$ & $100.55 \mathrm{ab}$ & 108.23 \\
& $\mathrm{G}$ & $0.45 \mathrm{~b}$ & $12.04 \mathrm{ab}$ & $76.70 \mathrm{ab}$ & 108.28 \\
NdeM-La & $\mathrm{Ch}$ & $7.28 \mathrm{ab}$ & $29.91 \mathrm{a}$ & $68.65 \mathrm{ab}$ & 72.07 \\
Resolana & $\mathrm{G}$ & $2.70 \mathrm{ab}$ & $18.93 \mathrm{ab}$ & $106.57 \mathrm{ab}$ & 72.48 \\
& $\mathrm{Ch}$ & $1.29 \mathrm{~b}$ & $2.04 \mathrm{~b}$ & $68.95 \mathrm{ab}$ & 82.59 \\
CP-62 & $\mathrm{G}$ & $1.59 \mathrm{ab}$ & $9.71 \mathrm{ab}$ & $106.52 \mathrm{ab}$ & 107.46 \\
& $\mathrm{Ch}$ & $0.77 \mathrm{~b}$ & $4.45 \mathrm{ab}$ & $105.59 \mathrm{ab}$ & 119.34 \\
CP-Centauro & $\mathrm{G}$ & $2.71 \mathrm{ab}$ & $3.68 \mathrm{ab}$ & $102.94 \mathrm{ab}$ & 110.58 \\
& $\mathrm{Ch}$ & $7.16 \mathrm{ab}$ & $11.43 \mathrm{ab}$ & $70.74 \mathrm{ab}$ & 66.13 \\
CP-Teloxtoc & $\mathrm{G}$ & $1.86 \mathrm{ab}$ & $18.14 \mathrm{ab}$ & $137.42 \mathrm{a}$ & 93.70 \\
& $\mathrm{Ch}$ & $0.64 \mathrm{~b}$ & $1.16 \mathrm{~b}$ & $74.63 \mathrm{ab}$ & 65.59 \\
CP-Ñañú1 & $\mathrm{G}$ & $1.19 \mathrm{~b}$ & $13.57 \mathrm{ab}$ & $115.01 \mathrm{ab}$ & 82.75 \\
& $\mathrm{Ch}$ & $1.98 \mathrm{ab}$ & $2.32 \mathrm{~b}$ & $29.04 \mathrm{~b}$ & 67.66 \\
CP-Mixe & $\mathrm{G}$ & $6.79 \mathrm{ab}$ & $9.95 \mathrm{ab}$ & $64.93 \mathrm{ab}$ & 88.73 \\
DSH (0.05) & $\mathrm{Ch}$ & $1.48 \mathrm{ab}$ & $4.38 \mathrm{ab}$ & $151.88 \mathrm{a}$ & 135.43 \\
Significancia & & 8.473 & 27.27 & 97.25 & 75.09 \\
\hline
\end{tabular}

Letras iguales por columna indican medias estadísticamente similares $(P \leq 0.05)$. dds: días después de la siembra, G: tamaño de cariópside grande, Ch: tamaño de cariópside chico, *: P $\leq 0.05$, DSH: diferencia significativa honesta, NS: no significativo.

$\mathrm{g}^{-1}$ (Cuadro 6); lo anterior difiere de lo reportado por Pérez et al. (2004), al mencionar que la AFE disminuye con la madurez; así mismo, que los valores de este índice pueden variar de acuerdo con factores como luz interceptada y edad de rebrote. Khurana y Singh (2000) relacionaron valores altos de AFE con TRC, debido a que asigna alta intercepción de luz y captura de carbono por unidad de masa interceptada, lo cual concuerda con lo encontrado en CP-Mixe CCh y CP-125 CG.
La TAN indica la cantidad de biomasa acumulada en función del área foliar; al respecto, Díaz-López et al. (2013) reportaron en maíz valores de $0.0102 \mathrm{~g} \mathrm{~m}^{-2} \mathrm{~d}^{-1}$ a los $34 \mathrm{dds}$, la cual posteriormente se redujo a $0.0018 \mathrm{~g} \mathrm{~m}^{-2} \mathrm{~d}^{-1}$ a los $63 \mathrm{dds}$; este comportamiento de valores altos en etapas iniciales se observó en el presente experimento (Cuadro 7), donde se presentaron diferencias significativas de 68 a 81 dds y el genotipo CP-Ñañú1 de CCh mostró un valor superior (0.0119 $\mathrm{g} \mathrm{cm}^{-2} \mathrm{~d}^{-1} ; \mathrm{P} \leq 0.01$ ); sin embargo, no fue diferente de CP-Teloxtoc CCh, NdeM-125 CG y NdeM-303 
Cuadro 7. Tasa de asimilación neta $\left(\mathrm{g} \mathrm{cm}^{-2} \mathrm{~d}^{-1}\right)$ en genotipos de Bouteloua curtipendula provenientes de diferente tamaño de cariópside y cuatro intervalos de muestreo, en condiciones de invernadero.

\begin{tabular}{|c|c|c|c|c|c|}
\hline \multirow{2}{*}{ Genotipos } & \multirow{2}{*}{ Tamaño de cariópside } & \multicolumn{4}{|c|}{ Intervalos de muestreo (dds) } \\
\hline & & $43-56$ & $57-67$ & $68-81$ & $82-96$ \\
\hline \multirow{2}{*}{ NdeM-125 } & G & 0.097 & 0.089 & $0.0055 \mathrm{ab}$ & 0.0019 \\
\hline & Ch & 0.217 & 0.088 & $0.0024 \quad b$ & 0.0008 \\
\hline \multirow{2}{*}{ NdeM-417 } & G & 0.060 & 0.010 & $0.0026 \mathrm{~b}$ & 0.0009 \\
\hline & $\mathrm{Ch}$ & 0.673 & 0.201 & $0.0041 \mathrm{~b}$ & 0.0008 \\
\hline \multirow{2}{*}{ NdeM-303 } & G & 0.534 & 0.050 & $0.0017 \quad b$ & 0.0014 \\
\hline & $\mathrm{Ch}$ & 0.075 & 0.018 & $0.0052 \mathrm{ab}$ & 0.0025 \\
\hline \multirow{2}{*}{ NdeM-La Resolana } & G & 0.090 & 0.023 & 0.0016 b & 0.0015 \\
\hline & Ch & 0.570 & 0.198 & $0.0038 \mathrm{~b}$ & 0.0019 \\
\hline \multirow{2}{*}{ CP-62 } & G & 0.252 & 0.031 & $0.0025 \mathrm{~b}$ & 0.0008 \\
\hline & $\mathrm{Ch}$ & 0.419 & 0.131 & $0.0040 \quad b$ & 0.0003 \\
\hline \multirow{2}{*}{ CP-Centauro } & $G$ & 0.151 & 0.060 & $0.0031 \mathrm{~b}$ & 0.0006 \\
\hline & $\mathrm{Ch}$ & 0.058 & 0.020 & $0.0041 \mathrm{~b}$ & 0.0009 \\
\hline \multirow{2}{*}{ CP-Teloxtoc } & G & 0.301 & 0.028 & 0.0016 b & 0.0006 \\
\hline & Ch & 0.548 & 0.258 & $0.0056 a b$ & 0.0014 \\
\hline \multirow{2}{*}{ CP-Ñañú1 } & G & 0.223 & 0.016 & $0.0014 \quad b$ & 0.0016 \\
\hline & $\mathrm{Ch}$ & 0.198 & 0.033 & $0.0119 a$ & 0.0027 \\
\hline \multirow{2}{*}{ CP-Mixe } & G & 0.118 & 0.009 & $0.0022 b$ & 0.0005 \\
\hline & Ch & 0.317 & 0.057 & $0.0027 \quad b$ & 0.0003 \\
\hline DSH (0.05) & & 0.7948 & 0.4133 & 0.002 & 0.0034 \\
\hline Significancia & & NS & NS & $\star *$ & NS \\
\hline
\end{tabular}

Letras iguales por columna indican medias estadísticamente similares $(\mathrm{P} \leq 0.05)$. dds: días después de la siembra, G: tamaño de cariópside grande, Ch: tamaño de cariópside chico, **: P $\leq 0.01, \mathrm{DSH}$ : diferencia significativa honesta, NS: no significativo.

cCh, lo cual indica que son los materiales con mayor aprovechamiento de la radiación solar; así mismo, puede indicar que estos materiales son capaces de producir mayor cantidad de materia seca debido a que la TAN indica eficiencia del tejido fotosintético, estos resultados son superiores a los señalados por Álvarez-Holguín et al. (2017), quienes reportaron valores de $0.00495 \mathrm{~g} \mathrm{~cm}^{-2} \mathrm{~d}^{-1}$ en ecotipos de pasto Banderita a los 70 dds y condiciones de invernadero.

\section{CONCLUSIONES}

NdeM-La Resolana con cariópside grande mostró mayor peso de inflorescencia desde $86 \mathrm{dds}$, lo que le confiere potencial para su establecimiento rápido en campo. NdeM-125 fue el único genotipo que no mostró diferencias para desarrollo vegetal por tamaño de cariópside. NdeM-La Resolana y CPMixe destacan en crecimiento para TACy AFE, respectivamente. La selección por tamaño de cariópside presenta potencial para lograr plántulas con mayor crecimiento temprano.

\section{AGRADECIMIENTO}

A CONACYT por el apoyo financiero brindado para el desarrollo de este trabajo a través de la beca de Maestría en Ciencias otorgada a la primera autora. Al proyecto de Problemas Nacionales Clave: 248252 "Colecta, Salvaguarda y Evaluación de Forrajeras (Poaceae) para Pastoreo Extensivo, Nativas de México Semiárido".

\section{BIBLIOGRAFÍA}

Álvarez-Holguín A., C. R. Morales-Nieto, R. Corrales-Lerma, J. S. SierraTristán y F. Villarreal-Guerrero (2017) Análisis del crecimiento de cinco genotipos de pasto banderita [Bouteloua curtipendula (Mich.) Torr.], bajo condiciones de invernadero. Tecnociencia Chihuahua 11:25-32.

Barrera J., D. Suárez y L. M. Melgarejo (2010) Análisis de crecimiento en plantas. In: Experimentos en Fisiología Vegetal. L. M. Melgarejo (ed.). Universidad Nacional de Colombia. Bogotá, Colombia. pp:25-38.

Castañeda-Saucedo M. C., C. López-Castañeda, M. T. B. Colinas-De León, J. C. Molina-Moreno y A. Hernández-Livera (2009) Rendimiento y calidad de la semilla de cebada y trigo en campo e invernadero. Interciencia 34:286-292. 
Corrales L. R., C. R. Morales N., A. Melgoza C., J. S. Sierra T., J. Á. Ortega G. y G. Méndez Z. (2016) Caracterización de variedades de pasto banderita [Bouteloua curtipendula (Michx.) Torr.] recomendadas para rehabilitación de pastizales. Revista Mexicana de Ciencias Pecuarias 7:201-211, https://doi.org/10.22319/rmcp. v7i2.4174

Cruz T. J. M., J. V. Ray R., J. L. Ledea R. y R. C. Arias P. (2017) Establecimiento de nuevas variedades de Cenchrus purpureus en un ecosistema frágil del valle del Cauto, Granma. Revista de Producción Animal 29:29-35

Di Benedetto A. y J. Tognetti (2016) Técnicas de análisis de crecimiento de plantas: su aplicación a cultivos intensivos. Revista de Investigaciones Agropecuarias 42:258-282.

Díaz-López E., J. M. Loeza-Corte, J. M. Campos-Pastelín, E. J. MoralesRosales, A. Domínguez-López y 0. Franco-Mora (2013) Eficiencia en el uso de la radiación, tasa de asimilación neta e integral térmica en función del fósforo en maíz (Zea mays L.). Agrociencia 47:135-146

Fernández R. J., M. Wang and J. F. Reynolds (2002) Do morphological changes mediate plant responses to water stress? A steadystate experiment with two $C_{4}$ grasses. New Phytologist 155:7988, https://doi.org/10.1046/j.1469-8137.2002.00438.x

García-Rodríguez J. J., M. A. Ávila-Perches, F. P. Gámez-Vázquez, M. De la 0-Olán y A. J. Gámez-Vázquez (2018) Calidad física y fisológica de semilla de maíz influenciada por el patrón de siembra de progenitores. Revista Fitotecnia Mexicana 41:31-37, https:// doi.org/10.35196/rfm.2018.1.31-37

Hernández-Guzmán F. J., A. R. Quero-Carrillo, P. Pérez-Rodríguez, M. Velázquez-Martínez y G. García-de los Santos (2015) Germinación y emergencia de propágulos de pasto en respuesta a pruebas de vigor. Revista Mexicana de Ciencias Agrícolas 6:1519-1532, https://doi.org/10.29312/remexca.v6i7.546

Hunt R. (2017) Growth analysis. Individual plants. In: Encyclopedia of Applied Plant Sciences. B. Thomas, B. G. Murray and D. J. Murphy (eds.). 2nd edition. Academic Press. London. pp:421429, https://doi.org/10.1016/B978-0-12-394807-6.00226-4

James J. J. (2008) Effect of soil nitrogen stress on the relative growth rate of annual and perennial grasses in the Intermountain West. Plant and Soil 310:201-210, https://doi.org/10.1007/s11104008-9645-X

James J. J. and R. E. Drenovsky (2007) A basis for relative growth rate differences between native and invasive forb seedlings. Rangeland Ecology and Management 60:395-400, https://doi. org/10.2111/1551-5028(2007)60[395:ABFRGR]2.0.CO;2

Khurana E. and J. S. Singh (2000) Influence of seed size on seedling growth of Albizia procera under different soil water levels. Annals of Botany 86:1185-1192, https://doi.org/10.1006/ anbo.2000.1288

Morales N. C. R., A. Quero C., A. Melgoza C., M. Martínez S. y P. Jurado G. (2009) Diversidad forrajera del pasto banderita [Bouteloua curtipendula (Michx.) Torr.], en poblaciones de zonas áridas y semiáridas de México. Técnica Pecuaria en México 47:231-244.

Pérez A. J. A., E. García M., J. F. Enríquez Q., A. R. Quero C., J. Pérez P. y A. Hernández G. (2004) Análisis de crecimiento, área foliar específica y concentración de nitrógeno en pasto "mulato" (Brachiaria hibrido, cv.). Técnica Pecuaria en México 42:447-458.

Peterson P. M., K. Romaschenko and Y. Herrera A. (2015) Phylogeny and subgeneric classification of Bouteloua with a new species, B. herrera-arrietae (Poaceae: Chloridoideae: Cynodonteae: Boutelouinae). Journal of Systematics and Evolution 53:351366, https://doi.org/10.1111/jse.12159

PMARP, Plan Maestro de la Alianza Regional para la Conservación de los Pastizales (2012) Plan Maestro de la Alianza Regional para la Conservación de los Pastizales del Desierto Chihuahuense 2011-2016. J. C. Guzmán-Aranda, J. Hoth y H. Berlanga (eds.) Comisión para la Cooperación Ambiental. Montreal, Canadá. 64 p.

Quero C. A. R., L. Miranda J. y J. F. Villanueva-Ávalos (2017a) Recursos genéticos de gramíneas para el pastoreo extensivo. Condición actual y urgencia de su conservación ante el cambio climático. Avances en Investigación Agropecuaria 21:63-85

Quero C. A. R., F. J. Hernández G., P. Pérez R., A. Hernández L., G. García S., P. Landa S. y S. E. Ramírez S. (2017b) Germinación de cariópsides clasificados por tamaño y diásporas de cuatro pastos para temporal semiárido. Revista Mexicana de Ciencias Agrícolas 8:489-502, https://doi.org/10.29312/remexca.v8i3.26

Quero-Carrillo A. R., L. Miranda-Jiménez, F. J. Hernández-Guzmán y F. A. Rubio A. (2014) Mejora del establecimiento de praderas de temporal. Folleto Técnico. Colegio de Postgraduados. Texcoco, Estado de México, México. 31 p, https://doi.org/10.13140/2.1.5101.2161

Quero-Carrillo A. R., F. J. Hernández-Guzmán, M. Velázquez-Martínez, H. G. Gámez-Vázquez, P. Landa-Salgado y P. Aguilar-López (2016) Métodos de establecimiento de pasturas en zonas áridas de México utilizando semillas crudas o cariópsides. Tropical Grasslands-Forrajes Tropicales 4:29-37, https://doi org/10.17138/TGFT(4)29-37

Reich P. B., C. Buschena, M. G. Tjoelker, K. Wrage, J. Knops, D. Tilman and J. L. Machado (2003) Variation in growth rate and ecophysiology among 34 grassland and savanna species under contrasting $N$ supply: a test of functional group differences. New Phytologist 157:617-631, https://doi.org/10.1046/j.14698137.2003.00703.x

Rincón A., G. A. Ligarreto y D. Sanjuanelo (2007) Crecimiento del maíz y los pastos (Brachiaria sp.) establecidos en monocultivo y asociados en suelos ácidos del piedemonte llanero colombiano. Agronomía Colombiana 25:264-272.

Sánchez-Arroyo J. F., C. Wehenkel, F. Ó. Carrete-Carreón, M. Murillo-Ortiz, E. Herrera-Torres and A. R. Quero-Carrillo (2018) Establishment attributes of Bouteloua curtipendula (Michx.) Torr. populations native to Mexico. Revista Fitotecnia Mexicana 41:237-243, https://doi.org/10.35196/rfm.2018.3.237-243

SAS Instutute (2009) SAS/STAT User's Guide Version 9.2. SAS Institute Inc. Cary, North Carolina, USA. 5136 p.

Smith K. F., N. M. McFarlane, V. M. Croft, P. J. Trigg and G. A. Kearney (2003) The effects of ploidy and seed mass on the emergence and early vigour of perennial ryegrass (Lolium perenne L.) cultivars. Australian Journal of Experimental Agriculture 43:481-486, ttps://doi.org/10.1071/EA01130 